\title{
Wie konnte es zur Krise im Rakhine-Staat in Myanmar kommen? Ein Blick zurück nach vorn
}

\author{
Mandy Fox $\cdot$ D. Hellmann-Rajanayagam $\cdot$ Rüdiger Korff
}

Eingegangen: 20. November 2020 / Angenommen: 29. Januar 2021

(C) Der/die Autor(en) 2021

Zusammenfassung Wie konnte es zu dem Konflikt im Rakhine-Staat in Myanmar kommen, der eine Flucht von mehr als 700.000 Muslim*innen nach Bangladesch zur Folge hatte? Um dieser Frage auf den Grund gehen zu können, ist es wichtig, die Ursachen und Hintergründe des Konfliktes zu betrachten und in den historischen Kontext einzubetten. Vielfältige Ebenen überlagern sich in dem Konflikt. Es ist zunächst ein lokaler Konflikt zwischen der muslimischen Minderheit und den buddhistischen Rakhine, desweiteren ein Konflikt zwischen dem Staat Myanmar, den Rakhine und den muslimischen Rohingya und letztlich ein internationaler Konflikt, der vor dem Internationalen Gerichtshof in Den Haag verhandelt wird.

Schlüsselwörter Rakhine · Rohingya · Arakan Army · Arakan Rohingya Salvation Army · Aung San Suu Kyi · Myanmar

Am 1. Februar 2021 putschte das Militär in Myanmar und unterbricht damit eine Dekade relativer Öffnung des Landes, sowie den Transitionsprozess hin zu einem demokratischen System. Das Manuskript dieses Essays wurde vor dem 1. Februar 2021 fertiggestellt und berücksichtigt somit die Ereignisse vor diesem Zeitpunkt.

M. Fox $(\bowtie) \cdot$ Prof. (em.) R. Korff

Universität Passau, Innstr. 33a, 94032 Passau, Deutschland

E-Mail: mandy.fox@uni-passau.de

Prof. (em.) R. Korff

E-Mail: ruediger.korff@uni-passau.de

Dr. D. Hellmann-Rajanayagam

Ludwig-Maximilians-Universität München, Geschwister-Scholl-Platz 1, München, Deutschland

E-Mail: daggi.rajanayagam@t-online.de 


\title{
How did the Crisis in Rakhine-State in Myanmar Come About? A Look Back to the Future
}

\begin{abstract}
What are the origins of the conflict in Rakhine State in Myanmar, a conflict that led to more than 700,000 Muslims fleeing to Bangladesh? To be able to investigate this question, it is essential to look at the causes and the background of the conflict and to put them into their historical context. The conflict has several overlapping levels. Firstly it is a local conflict between the Muslim minority and that of the Buddhist Rakhine, secondly one between the state, the Rakhine and the Muslim Rohingya and lastly an international conflict that is being heard at the International Court of Justice in The Hague.
\end{abstract}

Keywords Rakhine $\cdot$ Rohingya A Arakan Army $\cdot$ Arakan Rohingya Salvation Army · Aung San Suu Kyi · Myanmar

\section{Einleitung}

In Myanmar beansprucht der Konflikt im Rakhine-Staat ${ }^{1}$ besonders seit August 2017 die Aufmerksamkeit einer internationalen Öffentlichkeit, als mehr als 700.000 Muslim*innen, darunter Angehörige der Gruppe der Rohingya, von hier vor Kampfhandlungen flohen und seitdem in Camps in Bangladesch ausharren. Der Auslöser für die Flucht waren koordinierte Attacken auf myanmarische Grenzposten im Norden des Rakhine-Staates, verübt durch eine Rebellengruppe, die sich Arakan Rohingya Salvation Army (ARSA) nennt und von myanmarischer Regierungsseite als Terrorgruppe eingestuft wird. Bei der Attacke starben laut Regierungsangaben 59 Rebellen und 12 myanmarische Sicherheitskräfte. Das Militär reagierte mit Offensiven, die nicht nur Fluchtwellen von Muslim*innen nach Bangladesch zufolge hatten; auch Hindus, buddhistische Rakhine und andere Gruppen waren betroffen und flüchteten in südliche Regionen des Rakhine-Staates. ARSA, über die es nach wie vor wenig gesicherte Informationen gibt ${ }^{2}$, setzt sich laut eigenen Aussagen für die Rechte der muslimischen Rohingya ein und reklamiert auch den Angriff auf Grenzposten im Oktober 2016 für sich, in deren Folge der Norden schon einmal vom myanmarischen Militär abgeriegelt wurde und über 70.000 Menschen vor den Kampfhandlungen flohen (Fox 2017a).

Der Rakhine-Staat, im Westen Myanmars gelegen mit einer Grenze zu Bangladesch und von den übrigen Landesteilen Myanmars durch das von Nord nach

\footnotetext{
11974 wurde aus der Arakan-Region der Arakan-Staat und 1989 im Zuge der Umbenennung von Landes-, Städte-, und Straßennamen durch die burmesische Regierung der Rakhine-Staat.

2 ARSA, auch bekannt unter ihrem früheren Namen Harakah al-Yaqin, gründete sich offenbar 2013 als Reaktion auf die Vertreibungen der Muslim*innen im Rakhine-Staat im Jahr 2012. Sogenannte Anwerber trafen auf offene Türen, da besonders junge Menschen nun bereit waren, für ihre Rechte zu kämpfen. Geleitet wird ARSA laut der International Crisis Group von Rohingya, welche im Exil in Saudi-Arabien leben, finanzielle Unterstützung leisten und junge Rekruten in Nord-Rakhine in Guerillakriegstaktik ausbilden. Darüber hinaus gäbe es Pläne, ein Gebiet militärisch einzunehmen und Gebietsansprüche zu stellen, wie es andere bewaffnete ethnische Gruppen in Myanmar bereits getan haben (ICG 2016).
} 
Süd verlaufende Arakan Yoma Gebirge getrennt, befindet sich in einer Eskalationsspirale. Mit der Arakan Army (AA), die für mehr Autonomie des Rakhine-Staates kämpft, ist ein weiterer Akteur in den Konflikt eingetreten. Von der Regierung ebenfalls als terroristische Gruppe eingestuft, zählt die AA zwischen 5000 und 10.000 Kämpfern, vorwiegend buddhistische Rakhine, die sich besonders seit Januar 2019 Kämpfe mit dem Militär liefern. Die Konfliktzonen betreffen inzwischen auch andere Verwaltungsbezirke und nicht mehr nur den Norden des Rakhine-Staates. Der südliche Teil des Chin-Staates ist ebenfalls betroffen. Zwischen 60.000 und 160.000 Binnenvertriebene flüchteten, Stand Mai 2020, vor den Kämpfen (Fox 2020). Hinzu kommt, dass die Regierung neben einer strikten Kontrolle des Konfliktgebietes versucht, die Kommunikation massiv einzuschränken und so den Austausch von Informationen zu unterbinden. Die Regierung verhängte im Juni 2019 einen Shutdown für den mobilen Internetverkehr. Als Begründung gab das Ministerium für Verkehr und Kommunikation „Störungen des Friedens und die Nutzung von Internetdiensten zur Koordinierung illegaler Aktivitäten“ an (Amnesty International 2019, eigene Übersetzung). ${ }^{3}$

Die Frage, die sich unweigerlich stellt, ist: Wie konnte es zu diesem Konflikt und der Flucht von mehr als 700.000 Muslim*innen nach Bangladesch kommen? Um dieser Frage auf den Grund gehen zu können, ist es wichtig, die Ursachen und Hintergründe des Konfliktes zu betrachten und diese äußerst komplexe Situation in den historischen Kontext einzubetten. Ergänzend folgt ein Blick auf die Positionen der internationalen Gemeinschaft zu der Thematik sowie die daraus resultierenden Rückwirkungen auf die Situation in Myanmar. Eine Bewertung der aktuellen Lage vor Ort gestaltet sich schwierig, da der Zugang zum Konfliktgebiet und zu Informationen massiv eingeschränkt wird. Vielfältige Ebenen überlagern sich in diesem Konflikt. Es ist zunächst ein lokaler Konflikt zwischen der muslimischen Minderheit und der der buddhistischen Rakhine, desweiteren ein Konflikt zwischen dem Staat, den Rakhine und den muslimischen Rohingya und letztlich ein internationaler Konflikt, der vor dem Internationalen Gerichtshof (IGH) in Den Haag verhandelt wird. Die einzelnen Ebenen sind durch unterschiedliche Akteure mit ihren spezifischen Agenden geprägt und widersprechen sich teilweise, so dass eine komplexe Situation entsteht. Hinzu kommt, dass die Region seit Jahrzehnten chronisch unterforscht ist. Dies mag weniger an einem mangelnden Forschungsinteresse als an der Tatsache gelegen haben, dass es bis zur sogenannten Öffnung des Landes im Jahr 2011 kaum möglich war, in Myanmar und im Besonderen im Rakhine-Staat Forschung $\mathrm{zu}$ betreiben. ${ }^{4}$

\footnotetext{
${ }^{3}$ Siehe hierzu auch Robertson (2020). Treffen sollte der Internet-Blackout vor allem die AA. Im August 2020 erlaubte die Regierung 2G Services. 3G und 4G Services bleiben weiterhin ausgesetzt. Organisationen beklagen, dass das unzureichend ist (MDRF 2020).

${ }^{4}$ Für weitere Literatur siehe hierzu Jacques Leider, Bénédicte Brac de la Perrière, Pamela Gutman, HansBernd Zöllner.
} 


\section{Die dunkle Seite der Transition}

Die International Crisis Group (ICG) titelt in einem ihrer Berichte, dass die Gewalt gegen Muslim*innen in Myanmar die ,dunkle Seite der Transition“ sei (ICG 2013, eigene Übersetzung). Gewalt gegen Muslim*innen gab es bereits in vergangenen Jahrzehnten. Dass die Transition des Landes, eingeleitet durch die Wahlen im November 2010, eine entscheidende Bedingung für das Wiederaufflammen aktueller Konflikte darstellte, ist dennoch unbestritten. ${ }^{5}$ Das zeigt der Blick auf die vorherrschenden verfassungspolitischen Realitäten in Myanmar, die Situation im RakhineStaat sowie die verhärteten Positionen der am Konflikt beteiligten Gruppen.

\subsection{Verfassungspolitische Realitäten}

Die zivile Regierung Myanmars muss sich in einem unfreiwilligen politischen Tandem (Dyarchie) mit dem Militär arrangieren. Das Militär, welches in einem Topdown-Prozess eine Demokratisierung einleitete, verankerte in der Verfassung von 2008, dass über $25 \%$ der Parlamentssitze für dieses reserviert sind und nicht zur Wahl stehen. Qua Verfassung kontrolliert das Militär das Innenministerium, das Verteidigungsministerium und das Ministerium für Grenzangelegenheiten (Gerson 2017). Im November 2010 fanden die ersten Wahlen statt, die allerdings von ausländischen Beobachter*innen als weder frei noch fair eingeschätzt wurden (Lidauer 2012; Turnell 2011). Aus den Wahlen ging die durch das Militär aufgebaute Partei Union Solidarity and Development Party (USDP) als Sieger hervor. Unter Präsident Thein Sein, einem Ex-Militär, trieb die Regierung den politischen Reformprozess weiter voran, der am 8. November 2015 in weitgehend freien und fairen Wahlen mündete, bei denen die National League for Democracy (NLD) einen erdrutschartigen Sieg errang (Fox 2017b, 2020). Die Demokratiebewegung, u. a. repräsentiert durch die NLD, wurde unter der Militärdiktatur unterdrückt, vieles spielte sich im Verborgenen ab. Aktivist*innen und NLD-Anhänger*innen waren in der Vergangenheit als politische Gefangene inhaftiert. Demokratieerfahrungen aus der kurzen demokratischen Phase in den 1950er Jahren existieren für jüngeren Generationen nicht. Allerdings konnten sie in den letzten zehn Jahren der Öffnung des Landes ein freieres Leben führen. Mit dem Wahlsieg der NLD kamen neue Verantwortlichkeiten und Aufgaben, aber auch viel historischer Ballast und zahlreiche Herausforderungen. Staatsrätin und de-facto-Regierungschefin Aung San Suu Kyi und ihre Partei sehen sich konfrontiert mit über 20 bewaffneten ethnischen Gruppen, von denen einige seit über 60 Jahren für mehr Autonomie und Selbstbestimmung kämpfen, einem Staatsgebiet, das über weite Teile nicht durch die Regierung kontrolliert wird, 300.000 bis 500.000 Binnenvertriebenen, mehr als 1.000.000 Geflüchteten in den Nachbarländern sowie einem florierenden Drogenhandel, von dem sowohl Wider-

\footnotetext{
5 Im Jahr 2003 leitete die Militärjunta mit ihrer Roadmap to Democracy eine Demokratisierung zu ihren Bedingungen ein. Ein veranschlagtes Ziel nach dem anderen wurde abgearbeitet, darunter auch die Verabschiedung einer neuen Verfassung 2008.
} 
standsgruppen als auch die Armee profitieren. ${ }^{6}$ Das Ringen um Frieden stellt für die Entwicklung des multiethnischen Staates die größte Herausforderung dar. Darüber hinaus gilt es, den Wirtschaftssektor, das Bildungssystem und das Gesundheitswesen zu reformieren und die überbordende Korruption zu bekämpfen (Fox 2017b). Der Konflikt im Rakhine-Staat ist für die Zentralregierung somit nur einer von vielen in Myanmar und ein Ende der Gewaltspirale ist bislang nicht abzusehen.

\subsection{Die Situation im Rakhine-Staat}

Die Situation im Rakhine-Staat ist komplex, es können jedoch zwei Aspekte aus dieser vielschichtigen Gemengelage extrahiert werden: tiefsitzender Rassismus und Xenophobie sowie der Kampf um Ressourcen und Bürgerrechte. Die kurze demokratische Phase nach der Unabhängigkeit Burmas, wie sich das Land bis 1989 nannte, wurde 1962 durch einen Putsch von General Ne Win beendet. In der demokratischen Phase schien es, im Unterschied zu anderen Gebieten des Landes, noch keine großen Probleme mit Diskriminierung und Ausgrenzung der Muslim*innen in Rakhine gegeben zu haben. Die Rohingya wurden als ethnische Gruppe und Staatsbürger*innen bis Mitte der 1960er Jahre anerkannt. Das änderte sich nach dem Putsch. Der Slogan „Burmane sein, heißt Buddhist sein“ der Nationalismusbewegung, die während der britischen Kolonialzeit entstand, wurde wieder aufgegriffen. Robert Taylor (2015) bringt es auf den Punkt, wenn er schreibt, dass sich die Bamar, die Mehrheitsethnie im Land, gleich zweimal kolonisiert fühlte: zum einen durch die Briten und zum anderen durch Südasiat*innen. Burma war bis 1937 eine Provinz Indiens, was südasiatische Einwanderung und einen freien Kapitalfluss in die Kolonie bewirkte. Es wurde behauptet, dass der Buddhismus besonders durch das schnelle Wachstum der südasiatischen hinduistischen und muslimischen Bevölkerung gefährdet sei. Politische Aktivist*innen, darunter auch buddhistische Mönche, wiederholen diese Rhetorik auch heute noch (Taylor 2015). Xenophobie und ein latenter Rassismus wurden befördert und lassen sich auch an einem Zitat Ne Wins ablesen: „Even people of pure blood are being disloyal to the race. [...] If people of pure blood act this way, we must carefully watch people of mixed blood“ (zit. n. Gravers 1999, S. 69). Sowohl Chines*innen und Inder*innen, darunter Menschen muslimischen und hinduistischen Glaubens, als auch den Rohingya wurde vorgeworfen, illegale Immigrant*innen zu sein, die sich erst während der britischen Kolonialzeit in Burma niedergelassen hätten. Ausdruck all dessen waren u. a. das Staatsbürgerschaftsgesetz von 1982 und zwei militärische Operationen 1978 und 1991, die das Ziel hatten, illegale Migrant*innen im heutigen Rakhine-Staat aufzustöbern. Dies führte jeweils zu Fluchtwellen von einer Viertelmillion Muslim*innen nach Bangladesch.

\footnotetext{
${ }^{6}$ Das Myanmar Institute for Peace and Security (MIPS) spricht in seiner Annual Peace and Security Review 2020 für das Jahr 2019 und im Vergleich zu 2018 von einer Zunahme bewaffneter Zusammenstöße um $176 \%$. Grundlage für die Erhebung der Daten ist das Township-based Conflict Monitoring System. Vorrangig, so MIPS, geht es um zwei Konflikte: Den zwischen der AA und dem myanmarischen Militär (Tatmadaw) im nördlichen Rakhine- und südlichen Chin-Staat sowie zwischen der Ta'ang National Liberation Army und dem Militär im nördlichen Shan-Staat, die sich für $81 \%$ der bewaffneten Zusammenstöße verantwortlich zeichnen.
} 
Ethnizität wird instrumentalisiert und politisiert. Das burmanische Konzept von Ethnizität (thaing yin tha) geht davon aus, dass Menschen in einem Gebiet seit Anbeginn der Zeit leben. Muslim*innen oder Rohingya, so die Logik, sind keine indigenen, ethnischen oder nationalen Gruppen, da sie während der britischen Kolonialzeit eingewandert seien. Das Staatsbürgerschaftsgesetz von 1982 bestimmt die Staatsbürgerschaft anhand von Ethnie, Religion und Herkunft und macht Menschen zu Bürger*innen der ersten, zweiten und dritten Klasse. Die Regierung erkennt einige indigene Gruppen an, wie die Big Eight (Kachin, Kayah, Karen, Chin, Mon, Rakhine und Shan), also die sieben größten ethnischen Gemeinschaften nach denen auch die sieben Unionsstaaten benannt wurden und die Bamar, als Mehrheitsethnie im Land, die überwiegend in den sieben Regionen (divisions) lebt. Sie besitzen - auf dem Papier - die volle Staatsbürgerschaft, sind also Bürger der ersten Klasse. Die zweite Kategorie ist die assoziierte Staatsbürgerschaft (associated citizenship). Assoziierte Bürger*innen können die sein, deren Elternteile Bürger*innen im Sinne des Nationalitätengesetzes von 1948 waren. Bürger*in im Sinne dieses Gesetzes war jemand, dessen Vorfahren seit mindestens zwei Generationen permanent in Burma gelebt haben und dessen Eltern dort geboren waren. Es handelt sich somit um ältere Zuwander*innen aus der Kolonialzeit. Die dritte Kategorie sind die naturalisierten Bürger*innen (naturalized citizens), die vor dem 4. Januar 1948 in Burma geboren wurden, oder Eltern haben, die vor dem 4. Januar 1948 nach Burma gezogen sind, also jüngere Zuwander*innen der Kolonialzeit. Diese Bürger*innen haben jedoch das Problem, von dem Militär als potenzielles Sicherheitsrisiko angesehen zu werden (Yegar 2002, S. 62). Es wird durchaus unter Wissenschaftler*innen und Rechtsanwält*innen diskutiert, dass die Mehrheit der Rohingya laut Gesetz als assoziierte Bürger*innen gelten müsste (Kyaw 2017; Cheeseman 2017). Den Wortführern der Rohingya geht es jedoch nicht darum, sich als Zuwanderer der Kolonialzeit darzustellen, sondern um den Erhalt der vollen Staatsbürgerschaft, die die Anerkennung als ethnische Gruppe voraussetzt, oder den Nachweis, dass sie schon vor 1823, also vor dem ersten Anglo-Burmesischen Krieg, in Burma gelebt haben. Aufgrund dieser Bedingungen wird es daher wichtig, die eigenen Wurzeln zu belegen und sich in die Geschichte hineinzuschreiben, um u. a. politische Forderungen geltend zu machen. Die Verwendung des Namens Rohingya als Gruppenbezeichnung und seine versuchte historische Einbettung stellt ein großes Problem für die buddhistischen Rakhine und Bamar dar. Die Behauptung, die Rohingya seien eine ethnische Gruppe unter all den anderen ethnischen Gruppen in Myanmar mit weit zurückreichenden historischen Wurzeln, wird von den Rakhine und Bamar ebenso wenig akzeptiert wie die Forderung, unter ebendiesem Namen (Rohingya) die Staatsbürgerschaft zu fordern (Fox 2017a).

Ethnische Minderheiten, darunter auch die Rakhine, werfen der von Burman*innen dominierten Regierung und dem Militär vor, kein Mitspracherecht in eigenen Belangen zu haben, wenn es beispielsweise darum geht, sicherzustellen, dass sie von Investitionen profitieren, oder bezüglich Großprojekten, wie Staudämmen oder Bergbau, die die Menschen vor Ort massiv betreffen. ${ }^{7}$ Das spaltet die

\footnotetext{
7 Im Rahmen des chinesischen Projektes der neuen Seidenstraße (One Belt One Road Initative) sind viele solche Großprojekte auch im Rakhine vorgesehen, was ein Faktor für die Eskalation ist.
} 
Menschen in dem multiethnischen Staat eher, als dass es sie vereint. Es gibt Verteilungskämpfe, durch die Gruppen im Kampf um Privilegien, die beispielsweise durch das Militär bei Waffenstillstandsvereinbarungen in Aussicht gestellt werden, leicht gegeneinander ausgespielt werden können. Besonders die sieben Unionsstaaten sind reich an natürlichen Ressourcen und ein Zugriff darauf ist lukrativ (Fox 2017a). Dazu zählt auch der Rakhine-Staat, und besonders China ist an den Ressourcen interessiert. Allerdings ist der erstere auch einer der am wenigsten entwickelten in Myanmar. Die wirtschaftliche Lage ist schlecht, es gibt kaum Arbeitsmöglichkeiten und deshalb beständige Migrationsströme der Rakhine-Bevölkerung in andere Landesteile oder ins Ausland. Die Infrastruktur muss ausgebaut und das Bildungs- und Gesundheitssystem reformiert werden. Die Wahrnehmung des Rakhine-Staates als Krisenregion schreckt Investoren allerdings ab.

\subsection{Rohingya und buddhistische Rakhine}

Die Geschichte Arakans, des heutigen Rakhine-Staats, ist eng mit der Geschichte Bengalens verbunden. Seit Jahrhunderten gab es aufgrund der geographischen Gegebenheiten einen kulturellen und wirtschaftlichen Austausch zwischen Südasien und Südostasien. Diese kulturelle und religiöse Vielfalt im heutigen RakhineStaat stellte für die Militärregierung Myanmars keine Bereicherung, sondern eine Bedrohung ihres buddhistisch-burmanischen Staatskonzepts dar (South 2008; Leider 2002). Die Existenz von verschiedenen muslimischen Gruppen im RakhineStaat ist bis ins 15. Jahrhundert zurückzuverfolgen. Muslimische Händler könnte es bereits seit dem 9. Jahrhundert gegeben haben. Das Wort Rooinga heißt in der lokalen Sprache der Muslim*innen Rakhine und ist eine Herkunftsbezeichnung für Menschen aus der Rakhine-Region. Die politische Identität der Rohingya von heute wird allerdings jüngeren Entwicklungen zugeschrieben und soll sich in den 1940er Jahren herausgebildet haben (Tonkin 2014; Leider 2013; Zöllner 2008; Chan 2005). ${ }^{8}$ Genaue Aussagen darüber, wer die Rohingya von heute sind, wie viele es sind und wie sie sich selbst charakterisieren, können nicht getroffen werden, da es nach wie vor an Studien fehlt. Noch schwieriger wurde die Bestimmung nach dem Konflikt 2012, der die Identität umformte. Es war vorher weit verbreitet, dass die Muslim*innen von sich als ,Rakhine Muslime“9 ${ }^{\text {(6) }}$ sprachen, zumindest ist das bekannt für die Region Zentral-Rakhine. Seit 2012 sprechen die Binnenvertriebenen in den Camps, mehrheitlich Muslim*innen, von sich als Rohingya. Eine mögliche Interpretation könnte sein, dass es vor dem Konflikt keinen Grund gab, eine Identitätsbezeichnung einzufordern. Doch jetzt, wo sie ausgeschlossen sind, bedürfen sie dieser Gruppenidentität ${ }^{10}$ (Lewa 2003; Leider 2013).

\footnotetext{
${ }^{8}$ Curtis Lamprecht (2006) hingegen schreibt, dass das Wort Rohingya nicht der Politik entspringt, sondern ein historischer Name einer in Arakan (dem heutigen Rakhine) lebenden Gemeinde sei.

9 Bezeichnung entnommen aus den Interviews und der Feldforschung von Frau Fox aus den Jahren 20082020.

${ }^{10}$ Hierzu führte Frau Fox im Zuge ihrer Feldforschung auch ein persönliches Interview mit Herrn Lewa im Jahr 2013.
} 
Die buddhistischen Rakhine, die größte Gruppe im Rakhine-Staat ${ }^{11}$, berufen sich auf eine andere Version der Historie und sehen den Rakhine-Staat als die Wiege des Buddhismus. So legitimieren sie durch ihre Ursprungsmythen ihren Anspruch auf die Region. Meist aus dem Kontext gerissene historische Quellen sollen bezeugen, dass es die Gruppe der Rohingya noch nie in der Region Arakan gegeben hat. Sie seien illegale Einwander*innen aus Bangladesch mit dem Ziel, erst den Rakhine-Staat und dann ganz Myanmar zu islamisieren, d. h. die buddhistische Religion, Kultur und Identität zu zerstören (Fox 2017a). Solche Ängste und Überzeugungen zeigen sich bereits vor der sogenannten Öffnung des Landes 2011 und ziehen sich wie rote Fäden durch die vergangenen Jahrzehnte. Immer wieder wird die Angst geschürt, dass die Rohingya einen eigenen Staat fordern, wenn sie die Staatsbürgerschaft bekämen. Die neue Dimension, von einem vormals interkommunalen Konflikt im RakhineStaat (2012), nun zu einem bewaffneten Kampf von Rebellen, führt dazu, dass radikale buddhistische Nationalist*innen im übrigen Myanmar mit ihrer Propaganda auf offene Ohren stoßen und von der Krise profitieren. Die bekannteste buddhistische nationalistische Organisation ist die Vereinigung für den Schutz der Rasse und der Religion (MaBaTha), bestehend aus Mönchen, Nonnen und Laien. Sie schüren gezielt die Angst vor einer Islamisierung in Myanmar. Das erhöht das Konfliktpotential und gefährdet die Transformation des Landes. In den Gebieten, in denen der Konflikt im Jahr 2012 stattfand, werden Muslim*innen und Buddhist*innen immer noch gegeneinander abgeriegelt. Je separierter sie voneinander sind, desto größer die Entfremdung, desto schwieriger wird ein erneutes Zusammenleben und das Aufbauen von Vertrauen. Buddhistische Nationalist*innen nutzen diese Situation, um Ängste zu schüren und Feindbilder aufzubauen. Die Nutzung von Social Media unterstützt die Verbreitung von Hassreden und nationalistischen Narrativen (ICG 2017). Durch die als sehr einseitig und simplizistisch empfundene Darstellung internationaler Medienberichterstattung des Konflikts sowie die Erhöhung des internationalen Drucks auf die Regierung Myanmars, wächst der Zuspruch aus den Reihen der buddhistischen Nationalisten für das Militär und seinen Einsatz im Norden des RakhineStaates gegen die Rebellen. Insofern profitiert auch das Militär, denn es herrscht die Meinung, dass die staatliche Souveränität nur erhalten werden kann, wenn die Armee stark ist. Damit folgen sie der jahrzehntelangen Propaganda des Militärs, welches in der Bevölkerung vor nicht allzu langer Zeit rundweg abgelehnt worden ist.

\section{Reaktionen der Internationalen Gemeinschaft}

Im September 2018 kam eine Mission des UN-Menschenrechtsrates zu dem Schluss, dass in den Staaten Kachin, Rakhine und Shan Verbrechen gegen die Menschlich-

\footnotetext{
11 Entgegen des Eindrucks, dass im Rakhine-Staat nur buddhistische Rakhine und muslimische Rohingya leben würden, gibt es dort insgesamt sieben von der Regierung anerkannte ethnische Gruppen: (buddhistische) Rakhine, die wiederum die größte Gruppe bilden, Kwe Myi, Daingnet, Maramagyi, Mro, Thet, Kaman (Muslim*innen, die als ethnische Gruppe und Staatsbürger*innen anerkannt sind). Darüber hinaus leben Hindus im Rakhine-Staat, sowie Muslim*innen, die sich jedoch nicht alle zur Gruppe der Rohingya zählen.
} 
keit begangen wurden, hauptsächlich durch das myanmarische Militär, welches die Vorwürfe zurückweist (UNHRC 2018).

\subsection{Myanmar vor dem Internationalen Strafgerichtshof}

Der Staat Myanmar muss sich mittlerweile vor dem IGH verantworten, nachdem Gambia im Namen der 57-köpfigen Organisation für Islamische Zusammenarbeit im November 2019 eine Klage eingereicht hat. Myanmar wird die Verletzung der UN-Völkermordkonvention vorgeworfen (ICG 2019). Menschenrechtsorganisationen sprechen von Menschenrechtsverletzungen gegen die Gruppe der muslimischen Rohingya. Bilder und Augenzeugenberichte von Geflüchteten gingen um die Welt, die von Vergewaltigungen, Tötungen und abgebrannten Dörfern durch das myanmarische Militär zeugen (Fox 2017a). Dieses weist die Vorwürfe des Genocidal Intent ebenso zurück wie Staatsrätin und Außenministerin Suu Kyi, die höchstpersönlich nach Den Haag reiste, um die Sicht der myanmarischen Regierung und die Argumente der Verteidigung darzulegen. Die lange bestehende Furcht vor äußerer Einmischung in innere Angelegenheiten des Landes, vor Fremdbestimmung und Verlust der Souveränität - ein Trauma der Kolonialzeit - wurde in ihrer Rede deutlich und so verwundert ihre Aussage nicht: „Only if domestic accountability fails, may international justice come into play“ (zit. n. The Myanmar Times 2019). Die Anklage vor dem IGH wird besonders unter den Bamar als Angriff auf das gesamte Land wahrgenommen und löste eine Welle der Solidarität mit der Regierungspartei aus. Eine Verurteilung Myanmars durch den IGH würde jedoch noch keine unmittelbaren Maßnahmen zur Umsetzung nach sich ziehen. Zwar könnte Gambia den UN-Sicherheitsrat einschalten, welcher durch gezieltes Eingreifen die Durchsetzung des Urteils erwirken könnte; angesichts des Vetorechts der Volksrepublik China im UN-Sicherheitsrat erscheint ein solches Vorgehen gegen Myanmar jedoch äußerst unwahrscheinlich (Fox 2020).

Der IGH stellte im Januar 2020 in einer Vorentscheidung fest, dass Myanmar Sofortmaßnahmen zum Schutz der Minderheit der Rohingya ergreifen muss. Wegen der Coronakrise wurde die Frist bis Mai 2020 verlängert. Berichte von Menschenrechtsorganisationen stützen sich auf Aussagen Geflüchteter in den Lagern in Bangladesch und diese wiederum werden von sowohl der myanmarischen Regierung als auch durch das Militär angezweifelt. Eine UN-Fact-Finding-Mission lässt die Regierung im Rakhine-Staat jedoch nicht zu. Allenfalls bietet sie für Diplomat*innen und Journalist*innen je nach Sicherheitslage geführte Touren an, um sich vor Ort ein Bild der Lage zu machen. Offizielle Statements gibt es vom Militär und der Regierung, die jedoch schwer zu validieren sind, da der Zugang zum Konfliktgebiet strikt kontrolliert wird (Fox 2020).

\subsection{Empfehlungen der Advisory Commission on Rakhine State, Maßnahmen der EU und Deutschland}

Die Umsetzungen der Empfehlungen der Advisory Commission on Rakhine State, die vom ehemaligen UNO-Generalsekretär Kofi Annan geleitet und von Suu Kyi in Auftrag gegeben wurde, sind ebenfalls ins Stocken geraten. Zentrale Punkte, die 
in dem Bericht vom August 2017 ausführlich thematisiert werden, sind u. a. die Verbesserung der sozialen und wirtschaftlichen Lage breiter Bevölkerungsschichten sowie die Anerkennung der Diversität und der Identität der verschiedenen Gruppen und Gemeinschaften im Rakhine-Staat. An eine organisierte Rückführung und Garantien für die Sicherheit der Geflüchteten aus Bangladesch, wie in der UNHCRResolution im Juni 2020 gefordert, ist schon in Anbetracht der Kampfhandlungen zwischen dem Militär und der AA nicht zu denken, die wiederum neue Binnenvertriebene fordern. Trotz der möglichen Frustration der internationalen Gemeinschaft über die mangelnden Fortschritte lautet die Empfehlung der ICG: „Stay engaged““ (ICG 2020). Die Finanzierung von humanitärer Hilfe und Entwicklungszusammenarbeit sollte aufrecht erhalten werden. Ein Rückzug könnte sonst die strukturellen Faktoren verschärfen, die den zahlreichen Krisen in Myanmar zugrunde liegen. Ein problematischer Schritt ist die Ankündigung, dass sich Deutschland aus der bilateralen Entwicklungzusammenarbeit mit Myanmar zurückziehen wird. Bundesentwicklungsminister Gerd Müller (CSU) verkündete im Februar 2020 nach dem Besuch eines Flüchtlingslagers für Rohingya in Bangladesch, dass die Bundesregierung die Hilfe für Myanmar aussetzen wird, bis das Land die Rückkehr der Rohingya in Sicherheit garantiere. Nach dem neuen Strategiepapier Reformkonzept BMZ 2030, welches das Bundesministerium für wirtschaftliche Zusammenarbeit und Entwicklung (BMZ) im Mai 2020 vorstellte, ist klar, dass Myanmar zukünftig der Liste der direkt geförderten Länder entfällt. Ein fatales Signal an das Land und die zivilgesellschaftlichen Akteure, und ein Irrglaube, dass sich durch noch mehr Druck die Situation der Rohingya, geschweige denn eine Rückkehr ebendieser nach Myanmar, ändern würde.

Angesichts der vom Militär initiierten Übergriffe, die von der zivilen Regierung schwer kontrolliert werden können, führen die Maßnahmen der EU und Deutschlands zur Schwächung der zivilen, demokratischen Kräfte und zu einer internationalen Isolierung der myanmarischen zivilen Regierung. Die von der Europäischen Union im Februar 2018 beschlossenen Sanktionen gegen Myanmar angesichts der systematischen Menschenrechtsverletzungen wurden bis 30. April 2021 verlängert. Dazu gehören Einreiseverbote und Vermögenssperren gegen ranghohe Militärs, Exportbeschränkungen für Waffen und die Aussetzung der militärischen Zusammenarbeit. Kritische Stimmen mahnen, dass die internationale Gemeinschaft stattdessen den Dialog mit der Regierung und dem Militär suchen und die Zusammenarbeit verstärken sollte, um an einem zukunftsfähigen Plan zu arbeiten (Fox 2020).

\section{Ausblick}

Vor der Demokratisierung des Landes waren die Menschen in ihrer Ablehnung des Militärregimes vereint. Nun entfällt diese Verbindung und alte Konfliktlinien brechen wieder auf. Aus den vielen Ethnien soll eine nationale Identität geformt werden. Dies ist bisher nicht gelungen. Ideen von ethnischer Zugehörigkeit werden auch in Zukunft ein Motiv in der Politik Myanmars darstellen. Ein umfassenderes Verständnis der konzeptuellen Probleme, die die ethnische Zugehörigkeit für das Verständnis der Politik in Myanmar oder die Lösung von Menschenrechtsansprüchen aufwirft, 
so Taylor, würde dazu beitragen, ein inhärent emotionales Thema zu entpolitisieren. Die Frage der Rohingya ist letztendlich eine der Zugehörigkeit: Wer ist berechtigt, der Nation anzugehören, wer nicht, und vor allem: Wer hat das Recht, darüber zu entscheiden? Die Mehrheit der Bamar spricht jeglicher internationalen Organisation, aber auch ausländischen Individuen, das Recht ab, darüber zu befinden. Wer zur Nation gehört, ist ausschließlich die Angelegenheit der Bamar. Werden diese Wahrnehmungen nicht in die Beurteilung des Konfliktes und seine Lösungsansätze einbezogen, wird er sich noch lange hinziehen.

Funding Open Access funding enabled and organized by Projekt DEAL.

Open Access Dieser Artikel wird unter der Creative Commons Namensnennung 4.0 International Lizenz veröffentlicht, welche die Nutzung, Vervielfältigung, Bearbeitung, Verbreitung und Wiedergabe in jeglichem Medium und Format erlaubt, sofern Sie den/die ursprünglichen Autor(en) und die Quelle ordnungsgemäß nennen, einen Link zur Creative Commons Lizenz beifügen und angeben, ob Änderungen vorgenommen wurden.

Die in diesem Artikel enthaltenen Bilder und sonstiges Drittmaterial unterliegen ebenfalls der genannten Creative Commons Lizenz, sofern sich aus der Abbildungslegende nichts anderes ergibt. Sofern das betreffende Material nicht unter der genannten Creative Commons Lizenz steht und die betreffende Handlung nicht nach gesetzlichen Vorschriften erlaubt ist, ist für die oben aufgeführten Weiterverwendungen des Materials die Einwilligung des jeweiligen Rechteinhabers einzuholen.

Weitere Details zur Lizenz entnehmen Sie bitte der Lizenzinformation auf http://creativecommons.org/ licenses/by/4.0/deed.de.

\section{Literatur}

Amnesty International (2019). Myanmar end internet shutdown in Rakhine, Chin States. Amnesty International Public Statement. https://www.amnesty.org/en/documents/asa16/0604/2019/en/. Zugegriffen: 15. Jan. 2021.

Chan, A. (2005). The development of a Muslim enclave in Arakan (Rakhine) State of Burma (Myanmar). SOAS Bulletin of Burma Research, 3(2), 396-420.

Cheesman, N. (2017). How in Myanmar "national races" came to surpass citizenship and exclude Rohingya. Journal of Contemporary Asia, 47(3), 461-483.

Fox, M. (2017a). Die große Flucht. Südwind Magazin. https://www.suedwind-magazin.at/die-grosseflucht. Zugegriffen: 15. Jan. 2021.

Fox, M. (2017b). Blickwechsel: Ein Jahr NLD-geführte Regierung in Myanmar - Eine Bilanz. Stiftung Asienhaus. https://www.asienhaus.de/publikationen/detail/blickwechsel-ein-jahr-nld-gefuehrteregierung-in-myanmar-eine-bilanz/. Zugegriffen: 15. Jan. 2021.

Fox, M. (2020). Konfliktportrait Myanmar. Bundeszentrale für politische Bildung. https://www.bpb.de/ internationales/weltweit/innerstaatliche-konflikte/54586/birma-myanmar. Zugegriffen: 15. Jan. 2021.

Gerson, O.H. (2017). Sind Wahlen und eine Verfassung schon genug? Rechtsstaat und Rechtsstaatlichkeit in Myanmar unter der juristischen Lupe. Südostasien. https://crossasia-journals.ub.uni-heidelberg.de/ index.php/soa/article/view/1642/2369. Zugegriffen: 20. Okt. 2017.

Gravers, M. (1999). Nationalism as political paranoia in Burma. An essay on the historical practice of power. London: Routledge.

ICG (2016, 15. Dez.). Myanmar: a new Muslim insurgency in Rakhine State. Report $\mathrm{N}^{\circ}$ 283/Asia. https://www.crisisgroup.org/asia/south-east-asia/myanmar/283-myanmar-new-muslim-insurgencyrakhine-state. Zugegriffen: 15. Jan. 2021.

ICG (2017, 5. Sept.). Buddhism and state power in Myanmar. Asia Report $\mathrm{N}^{\circ} 290$. https://www.crisisgroup. org/asia/south-east-asia/myanmar/290-buddhism-and-state-power-Myanmar. Zugegriffen: 15. Jan. 2021.

ICG (2019, 10. Dez.). Myanmar at the International Court of Justice. https://www.crisisgroup.org/asia/ south-east-asia/myanmar/myanmar-international-court-justice. Zugegriffen: 15. Jan. 2021. 
ICG (2020, 27. Mai). International Crisis Group Watch List 2020 Spring Edition. https://www.crisisgroup. org/global/watch-list-2020-spring-edition. Zugegriffen: 10. Juni 2020.

ICG - International Crisis Group (2013). The dark side of transition: violence against Muslims in Myanmar. Report $\mathrm{N}^{\circ}$ 251/Asia. https:/www.crisisgroup.org/asia/south-east-asia/myanmar/dark-sidetransition-violence-against-muslims-myanmar. Zugegriffen: 15. Jan. 2021.

Kyaw, N. N. (2017). Unpacking the presumed statelessness of Rohingyas. Journal of Immigrant \& Refugee Studies, 15(3), 269-286.

Lamprecht, C. (2006). Burma (Myanmar). In G. Fealy, \& V. Hooker (Hrsg.), Voices of Islam in Southeast Asia. A contemporary sourcebook (S. 23-29). Singapore: ISEAS.

Leider, J.P. (2002). On Arakanese territorial expansion. Origins, context, means and practice. In J. Gommens, \& J. Leider (Hrsg.), The maritime frontier of Burma: exploring political, cultural and commercial interaction in the Indian Ocean World, 1200-1800 (S. 127-150). Leiden: KITLV Press.

Leider, J. P. (2013). Rohingya. The name, the movement, the quest for identity. Nation building in Myanmar. Yangon, Myanmar Egress \& Myanmar Peace Center. http://www.networkmyanmar.org/ESW/ Files/Leider-Rohingya.pdf. Zugegriffen: 7. Apr. 2021.

Lewa, C. (2003). Conflict, discrimination and humanitarian challenges in Northern Arakan State. https:// www.burmalibrary.org/sites/burmalibrary.org/files/obl/docs/Chris-EU_Paper.htm. Zugegriffen: 8. Mai 2008.

Lidauer, M. (2012). Democratic dawn? Civil society and elections in Myanmar 2010-2012. Journal of Current Southeast Asian Affairs, 31(2), 87-114.

MDRF - Myanmar Centre for Responsible Business (2020). MDRF calls for the end of the 2G digital blackout. https://www.myanmar-responsiblebusiness.org/news/mdrf-calls-for-end-of-blackout.html. Zugegriffen: 15. Jan. 2021.

Robertson, P. (2020). Myanmar again cuts Rakhine states's internet. Human Rights Watch. https://www. hrw.org/news/2020/02/05/myanmar-again-cuts-rakhine-states-internet. Zugegriffen: 15. Jan. 2021.

South, A. (2008). Ethnic politics in Burma. States of conflict. New York: Routledge.

Taylor, R.H. (2015). Refighting old battles, compounding misconceptions: the politics of ethnicity in Myanmar today. ISEAS Perspective, 12, 1-16.

The Myanmar Times (2019, 11. Dez.). Daw Aung San Suu Kyi's speech in full. https://www.mmtimes. com/news/daw-aung-san-suu-kyis-icj-speech-full.html. Zugegriffen: 15. Jan. 2021.

Tonkin, D. (2014). The Rohingya identity. British Experience in Arakan 1826-1948. www.networkmyanmar. org. Zugegriffen: 9. Apr. 2014.

Turnell, S. (2011). Myanmar in 2010: doors open, doors close. Asian Survey, 51(1), 148-154.

UNHRC - United Nations Human Rights Council (2018). Report of the independent international factfinding mission on Myanmar. A/HRC/39/64. https://www.ohchr.org/en/hrbodies/hrc/myanmarFFM/ Pages/ReportoftheMyanmarFFM.aspx. Zugegriffen: 15. Jan. 2021.

Yegar, M. (2002). Between integration and secession - the Muslim communities of the Southern Philippines, Southern Thailand and Western Burma/Myanmar. Lanham: Lexington Books.

Zöllner, H.-B. (2008). Die Rohingyas - Konstruktion, De-Konstruktion und Re-Konstruktion einer ethnisch-religiösen nationalen Identität. Austrian Journal of South-East Asian Studies, 1(1), 53-64. 